\title{
Photoelectrical Characteristics of UV Organic Thin-film Transistor Detectors
}

\author{
Dao Thanh Toan* \\ Faculty of Electrical-Electronic Engineering, University of Transport and Communications, \\ No.3, Cau Giay, Dong Da, Hanoi, Vietnam \\ Received 16 March 2017 \\ Revised 05 May 2017; Accepted 30 May 2017
}

\begin{abstract}
In this paper, a pentacene photo organic thin-film transistor (photoOTFT) was fabricated and characterized. The gate dielectric acted as a sensing layer thanks to it strongly absorbs UV light. Electrical behaviors of photoOTFT were measured under $365 \mathrm{~nm}$ UV illumination from the gate electrode side. The current in transistor channel was significantly enhanced by photoelectrons at interface of buffer/gate dielectric. Photosensitivity increased with the light intensity but decreased with the applied gate voltage. Meanwhile the photoresponsivity decreased with the light intensity and increased with the applied gate voltage. The transistor responses well with the pulse of light with many test cycles of light-on and light-off. The best photosensitivity, photoresponsivity, rising time and falling time parameters of the device were found to be about $10^{4}, 0.12 \mathrm{~A} / \mathrm{W}$, and $0.2 \mathrm{~s}$, respectively. The obtained photoelectrical results suggest that the photoOTFT can be a good candidate for practical uses in low-cost UV optoelectronics.
\end{abstract}

Keywords: Pentacene phototransistor, UV sensor, organic electronics, optoelectronics.

\section{Introduction}

In recent years, electronic components manufacturing from organic materials have been intensively studied due to their modern applications of low-cost, flexible, large area, lightweight lighting, and bendable display, which are hard to be realized using conventional inorganic semiconductors [1-5]. Evidently, an OLED Television has been succeeded to enter in the market and the OLED technology is going to occupy in all displays of the modern electronic products. Besides OLED, photodetection device operating in the ultraviolet (UV) region are increasingly attracting attention due to a wide variety of potential applications, such as water purification, sterilization, medicine, fire alarm, ozone sensing, a solar UV radiation monitor, or organic visible light communication [6-15]. In recent work [14], we have proposed a new approach to construct a UV photo pentacence OTFT (organic thin-film transistor) via introducing the photoactive molecules of

\footnotetext{
*Corresponding author. Tel.: 84-979379099.

Email: daotoan@utc.edu.vn

https://doi.org/10.25073/2588-1124/vnumap.4077
} 
DPA-CM (6-[4'-(N,N-diphenylamino)phenyl]-3-ethoxycarbonylcoumarin) doped in gate dielectric polymer of PMMA (poly(methyl methacrylate)). The operation mechanism study realized that the DPA-CM act as an UV light sensing material that is potential to overcome the limitation of mismatching the absorption wavelength of the semiconducting material of pentacene with the UV light wavelength. However, in order to make the transistor device enable for an application in optoelectronics, the critical photodevice parameters of photosensitivity, response time, and photoresponsivity of the photoOTFT are necessary to be investigated [5].

In the present work, a photoOFFT with a pentacene semiconductor and a photoactive gate dielectric is re-fabricated. Then, the photoelectrical characteristics at different applied voltages and light intensities are measured to estimate the device parameters. The photodevice exhibits a high photosensitivity or photoresponsivity and fast response characteristic.

\section{Experimental methods}

(a)

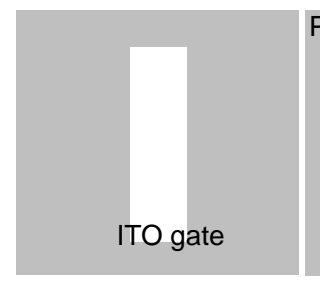

(b)

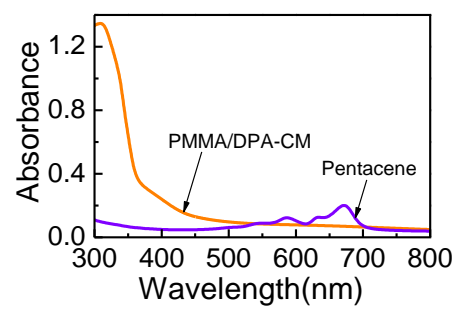

(c)

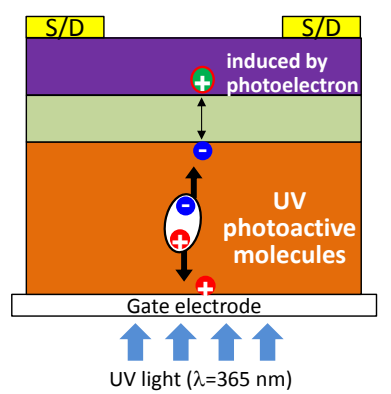

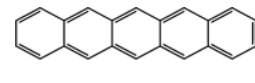

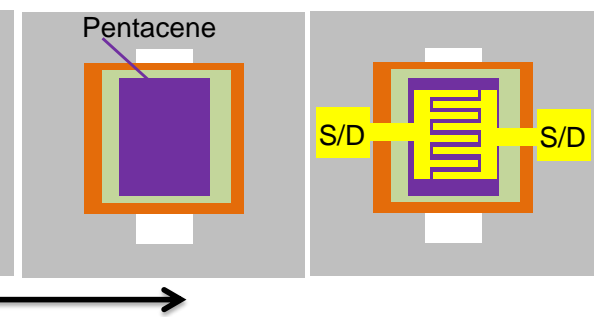

(d)
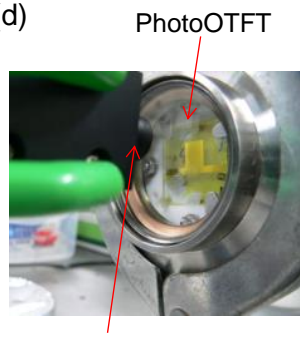

Head of UV light source

Fig. 1. a, Fabrication process of photoOTFT. S/D stands for source/drain electrodes. Arrow is to indicate process. $b$, Absorbance spectra of photoactive dielectric of PMMA/DPA-CM and pentacene measured using JASCO V-570 spectrometer. c, Illustration of cross-sectional structure and UV light irradiation method. d, Camera image of fabricated photoOTFT under test.

The photoOTFT was fabricated by employing the previous method [14]. The fabrication process, device structure and the properties of the main materials are shown in Fig. 1. Firstly, glass substrates coated with a $150 \mathrm{~nm}$ gate electrode layer of indium tin oxide (ITO) were cleaned using ultrasonication, followed by $\mathrm{UV}-\mathrm{O}_{3}$ treatment. PMMA and DPA-CM were dissolved in chloroform at 
a concentration 2 wt \%. Absorbance spectra of the PMMA/DPA-CM thin-film on quartz are shown in Fig. 1b. A 260-nm-thick photoactive dielectric layer of the PMMA/DPA-CM was prepared by spincoating and heated on a hot plate at $100{ }^{\circ} \mathrm{C}$ for $60 \mathrm{~min}$ to remove the residual solvent. Next, a $\sim 70-\mathrm{nm}$ thick polystyrene (Aldrich, $M_{\mathrm{w}}=280,000$ ) buffer layer was formed onto the PMMA/DPA-CM layer by spin-coating of a m-xylene solution $(1 \mathrm{wt} \%)$ at $1000 \mathrm{rpm}$ for $60 \mathrm{~s}$ and dried at $100{ }^{\circ} \mathrm{C}$ for $60 \mathrm{~min}$. The buffer layer here is needed to avoid chemical doping on the semiconducting channel by the photoelectrons from the photoactive dielectrics. Subsequently, a 50-nm-thick layer of pentacene (Aldrich, purified by vacuum sublimation twice) was formed on the buffer layer by vacuum deposition at a deposition rate of $0.02 \mathrm{~nm} \mathrm{~s}^{-1}$. Finally, the device was completed by deposition of 50-nm-thick source-drain electrode of gold at a deposition rate of $0.03 \mathrm{~nm} \mathrm{~s}^{-1}$ through a designed metal mask to form the length $(L)$ and width $(W)$ of the channel of 50 and $2000 \mu \mathrm{m}$, respectively. The all vacuum deposition processes were done at a pressure of $2 \times 10^{-6}$ Torr.

The thickness of thin film was checked by scratching the film and measuring a height difference across the scratch with an atomic force microscope (VN-8000, KEYENCE). Electrical measurements of the photoOTFT were performed using a Keithley 4200 semiconductor characterization system in a dry nitrogen atmosphere at room temperature. $365 \mathrm{~nm}$ UV light generated from an Omron ZUV UV irradiator was irradiated from a glass substrate side as presented in Figs. 1c and 1d.

\section{Results and discussion}

Black curves in Fig. 2 present the electrical characteristics of the initial photoOTFT. Regarding basic transistor device performance, the saturation-region hole mobility $(\mu)$ is estimated by fitting the plot of the square root of drain current $\left(I_{\mathrm{D}}\right)$ versus gate voltage $\left(V_{\mathrm{G}}\right)$ with an equation [14]:

$$
I_{D}=\frac{W}{2 L} C_{i} \mu\left(V_{G}-V_{t h}\right)^{2},
$$

where $V_{\text {th }}$ is the threshold voltage. The $V_{\text {th }}$, on/off current ratio, $\mu$, and swing factor, estimated from the transfer characteristics at a drain voltage $\left(V_{\mathrm{D}}\right)$ of $-5 \mathrm{~V}$ shown in Fig. $2 \mathrm{~b}$, are $-3.45 \mathrm{~V}, 3.48 \times 10^{5}$, and $0.025 \mathrm{~cm}^{2} \mathrm{~V}^{-1} \mathrm{~s}^{-1}, 1.22 \mathrm{~V} /$ decade, respectively. The similarity of the initial characteristics to other organic transistors [1,9-14] confirming that the fabrication process is proper.

The differences in both transfer and output curves under dark, light, and after light-off shown in Fig. 2 clearly indicate a UV photo sensing property of the fabricated device. As shown in Fig. 1b, the absorbance of pentacene at $365 \mathrm{~nm}$ is very weak, leading to a photocurrent originating from direct carrier generation in pentacene is negligible. On other aspect, the absorbance of the PMMA/DPA-CM is much stronger than that of pentacene in the UV region, resulting in the DPA-CM plays an important role to construct the UV pentacene photoOTFT. The working principle was detailed in our previous report [14], here it is briefly explained. When the photoOTFT is irradiated and biased. The chargeseparation state is generated and under the application of a voltage between the gate and drain electrodes, the charge-separation state is converted into free electrons and holes. Under effect of the gate electric field, the photogenerated holes move to the ITO gate electrode and the photogenerated electrons move to the interface of the photoactive/polystyrene buffer layers. The additional electric field made by the photogenerated electrons further induces additional holes accumulation in the pentacene transistor channel (see Fig. 1(c)). As the result, the concentration of hole in the channel becomes larger than that of the device in dark, leading to the increasing the $I_{\mathrm{D}}$ as indicated by red curves in Fig. 2. On the other hand, when the UV light is removed, the PMMA/DPA-CM and buffer 
layer work as a normal gate dielectric layer for field-effect operation and thus hole accumulation in the transistor channel is inducted by the gate electric field only, leading to decreasing the $I_{\mathrm{D}}$ as presented by black curves in Fig. 2 .
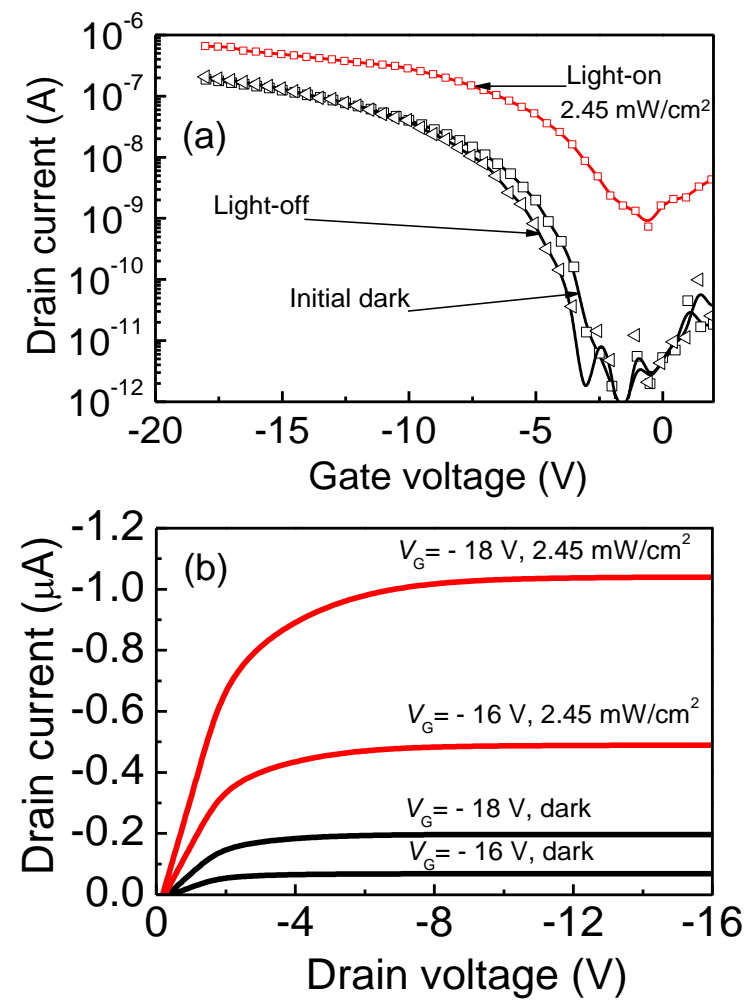

Fig. 2. Transfer (a) and output (b) characteristics of photoOFET under dark and UV light of $2.45 \mathrm{~mW} / \mathrm{cm}^{2}$.

The photosensitivity $P$ of the phototransistor can be estimated by the following equation [5]:

$$
P=\frac{I_{D, i l l}-I_{D, \text { dark }}}{I_{D, \text { dark }}}
$$

where $I_{\mathrm{D}, \text { ill }}$ and $I_{\mathrm{D}, \text { dark }}$ are the drain current under light illumination and dark, respectively. Figure $3 \mathrm{a}$ shows the relationship between the $P$ and gate voltage at different light intensities. The $P$ tends to decrease with applied gate voltage, and almost saturates at UV light intensity higher than 25.60 $\mathrm{mW} / \mathrm{cm}^{2}$. This tendency is similar to the experimental data reported by other groups [12-14]. The maximum $P$ is realized at a $V_{\mathrm{G}}=-2 \mathrm{~V}$ as indicated by dotted line in Fig. 3a. Fig. 3b plotted the maximum $P$ versus light intensity. As can be seen, the large maximum $P$ is obtained to be from $10^{3}$ to $10^{4}$ corresponding to increasing light intensity.

The $R$ of the photoOTFT is determined by formula [15]:

$$
R=\frac{I_{D, i l l}-I_{D, d a r k}}{P_{o p t} \times A}
$$

where $P_{\text {opt }}$ is the incident light intensity, $A$ is the area of the transistor channel, which can be calculated by $\mathrm{W} \times \mathrm{L}$. Thus, eq (3) can be converted to be: 


$$
R=\frac{I_{D, i l l}-I_{D, \text { dark }}}{P_{\text {opt }} \times W \times L}
$$

Using eq (4) and based on the experimental data, the calculated $R$ is presented in Fig. 4. Unlike $P$, the $R$ was found to increase as increasing the applied $V_{\mathrm{G}}$. Also, dependence of the $R$ on the UV light intensity has been summarized. As shown in Fig. $4 \mathrm{~b}$, the $R$ decreased as increasing the light intensity. At a certain intensity of light, the maximum $R\left(R_{\max }\right)$ was obtained to be in a range of $0.01-0.1 \mathrm{~A} / \mathrm{W}$.

Besides the $P$ and the $R$, the response time is other important parameter of the photodevice. Figure 5 shows the response time of the photoOTFT at UV light power of $2.45 \mathrm{~mW} / \mathrm{cm}^{2}$ recording by a digital oscilloscope. The $V_{\mathrm{G}}$ of $-2 \mathrm{~V}$ was chosen since at this value, the photodevice can reach the maximum $P$ as mentioned above. As shown, the repeatable change in the drain current is well correspondent to the cycle of the light-on and light-off. Utilizing the response behavior, the rising time $\left(\tau_{\text {rise }}\right)$ and falling time $\left(\tau_{\text {fall }}\right)$ were measured to be about $0.2 \mathrm{~ms}$, indicating that the device has a fast response property in comparison with that in the recent pentacene phototransistor [10].
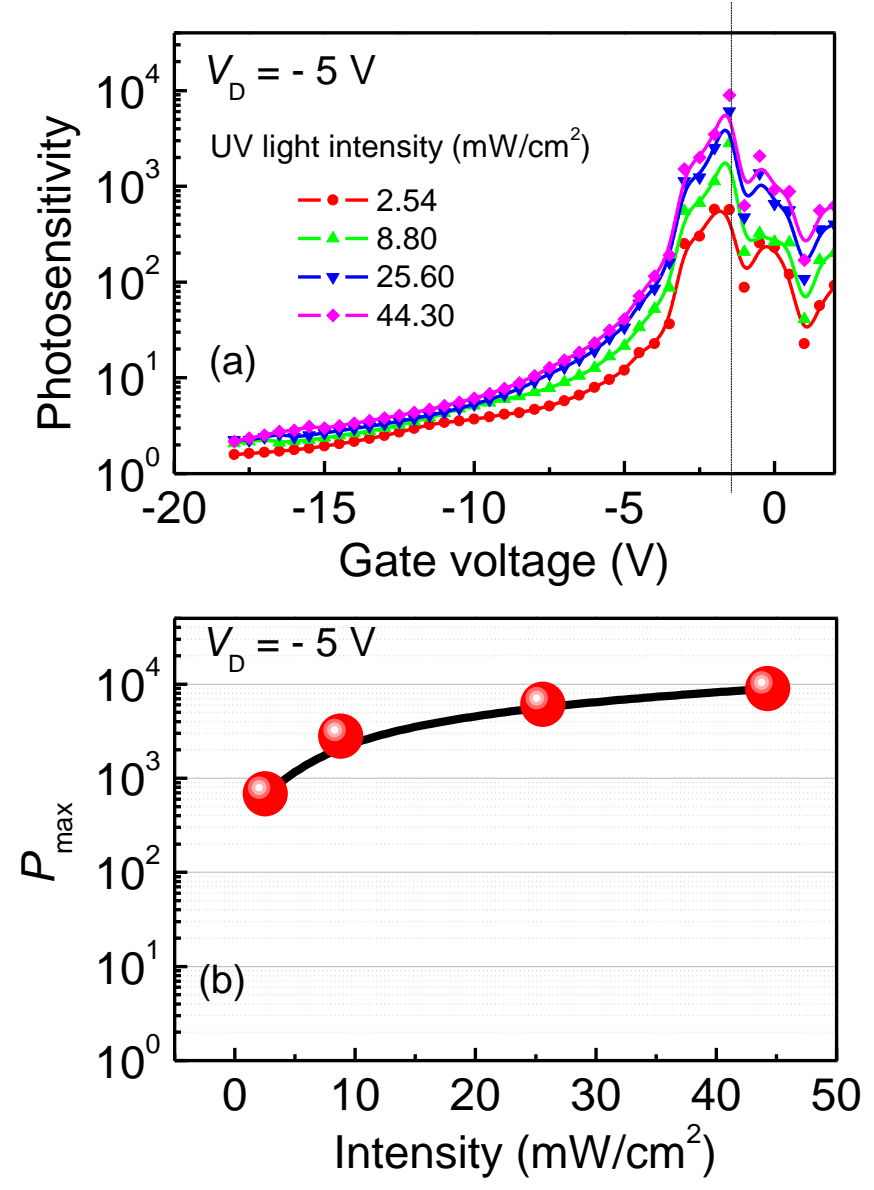

Fig. 3. Photosensitivity versus gate voltage at various UV light intensities (a) and maximum photosensivity versus intensity (b) of fabricated photoOTFT at $V_{\mathrm{D}}$ of $-5 \mathrm{~V}$. 

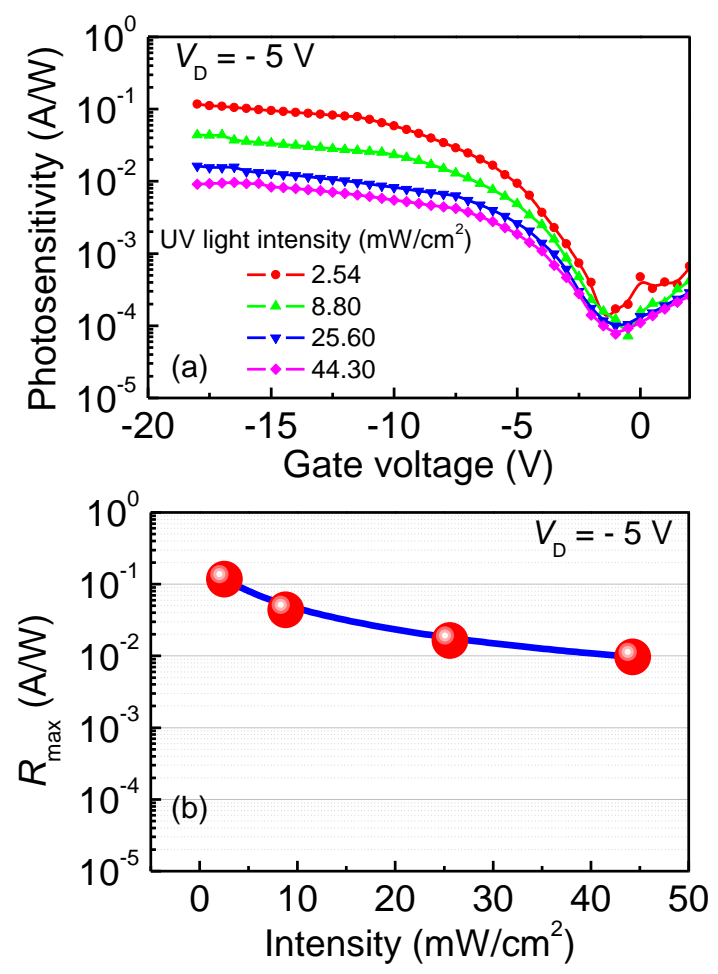

Fig. 4. (a) Relationship between photoresponsivity and gate voltage at different UV light intensities and (b) maximum photoresponsivity as function of intensity of fabricated pentacene photoOTFT at $V_{\mathrm{D}}$ of $-5 \mathrm{~V}$.
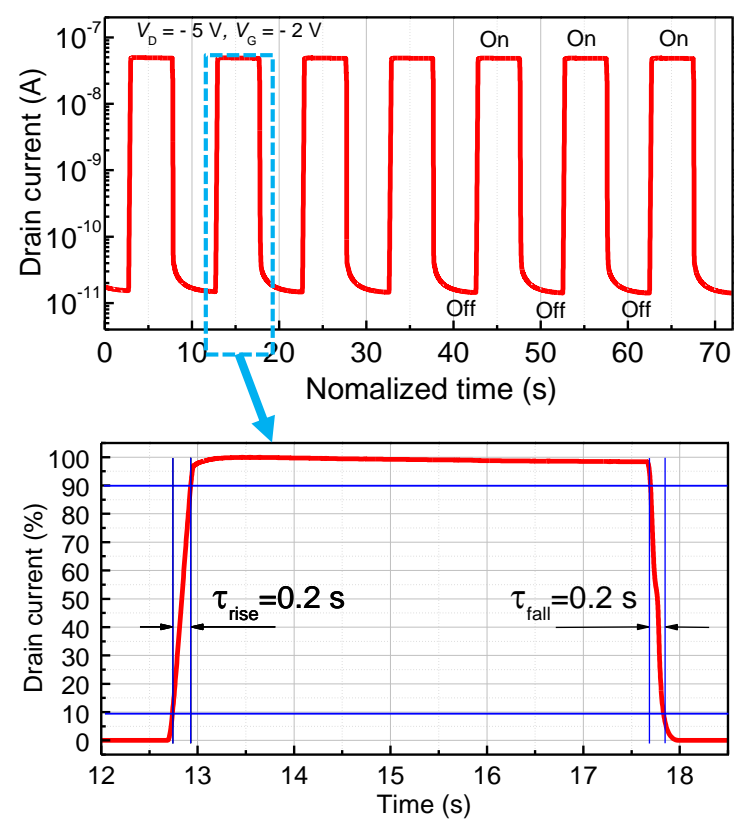

Fig. 5. (Top) Response time characteristics and (Bottom) determinations of rising time and falling time of pentance photoOTFT. 
Table 1 presents the summary of device performance of the present pentacene photoOTFT and relative works reported so far in terms of operating wavelength $(\lambda)$, channel area, $\tau_{\text {rise }}, \tau_{\text {fall }}$, maximum $P$, and maximum $R$. The $R$ is current device approaches the commercial value and can be comparable to that in other pentacene based-OTFTs. Significantly, the pentacene photoOTFT shows advancement with respect to the $\tau_{\text {rise }}, \tau_{\text {fall }}, P$. This is due to the fact that the photoOTFT was designed and made using a different approach, where the gate dielectric works as a UV sensing layer.

Table 1. Summary of device performance of current photoOTFT and relative photodevices.

\begin{tabular}{|c|c|c|c|c|c|c|c|}
\hline UV sensor & $\begin{array}{l}\lambda \\
(\mathrm{nm})\end{array}$ & $\begin{array}{l}\mathrm{L} \times \mathrm{W} \\
(\mu \mathrm{m} \times \mu \mathrm{m})\end{array}$ & $\begin{array}{l}\tau_{\text {rise }} \\
(\mathrm{s})\end{array}$ & $\begin{array}{l}\tau_{\text {fall }} \\
(\mathrm{s})\end{array}$ & $\mathrm{P}$ & $\begin{array}{l}\mathrm{R} \\
(\mathrm{A} / \mathrm{W})\end{array}$ & Year, Ref \\
\hline Inorganic $\mathrm{SiC}$ & $210-380$ & N/A & N/A & N/A & N/A & 0.13 & Industry, Ref. 6 \\
\hline $\begin{array}{l}\text { Pentacene } \\
\text { OTFT }\end{array}$ & 364 & $90 \times 500$ & N/A & N/A & $\sim 1.0 \times 10^{1}$ & N/A & 2006, Ref. 9 \\
\hline $\begin{array}{l}\text { Pentacene } \\
\text { OTFT }\end{array}$ & 365 & $50 \times 4000$ & 60 & 60 & $\sim 1.0 \times 10^{4}$ & 0.015 & 2009, Ref. 10 \\
\hline $\begin{array}{l}\text { Pentacene } \\
\text { OTFT }\end{array}$ & 365 & $100 \times 4000$ & N/A & N/A & $\sim 1.2 \times 10^{4}$ & 0.07 & 2012, Ref. 12 \\
\hline $\begin{array}{l}\text { Pentacene } \\
\text { OTFT }\end{array}$ & 350 & $100 \times 17200$ & N/A & N/A & $\sim 1.0 \times 10^{4}$ & 0.08 & 2013, Ref. 13 \\
\hline $\begin{array}{l}\text { Pentacene } \\
\text { OTFT }\end{array}$ & 365 & $50 \times 2000$ & 0.2 & 0.2 & $\sim 1.0 \times 10^{4}$ & 0.12 & Current work \\
\hline
\end{tabular}

\section{Conclusions}

In conclusion, an UV pentacene photoOTFT with a sensing layer of gate dielectric has been fabricated and characterized. Electrical behaviors of phototransistor were investigated at $365 \mathrm{~nm} \mathrm{UV}$ irradiation from the gate electrode side. The enhancement of the photocurrent in transistor channel resulted from the photoelectrons at the buffer/gate dielectric interface. Photosensitivity was found to increase with the light intensity and decrease with the $V_{\mathrm{G}}$. On contrast, the photoresponsivity was observed to decrease with the light power and increase with the $V_{\mathrm{G}}$. The pentecene transistor rapidly responded with the light-on and light-off. The highest photosensitivity, largest photoresponsivity, fastest rising/falling time of the phototransistors were recorded to be $10^{4}, 0.12 \mathrm{~A} / \mathrm{W}$, and $0.2 \mathrm{~s}$, respectively. Such photoelectrical data indicate that the fabricated photoOTFT is highly potential for practical low-cost UV optoelectronic circuits.

\section{Acknowledgements}

Author would like to thank the International Information Science Foundation, 2016, Tokyo, Japan (grant no. 2016.1.3.126) and Prof. H. Sakai, JAIST, Japan for supporting facilities of semiconductor component manufacturing. 


\section{References}

[1] R. Liguori , W.C. Sheets, A. Facchetti, A. Rubino, Light- and bias-induced effects in pentacene-based thin film phototransistors with a photocurable polymer dielectric, Organic Electronics, 28 (2016) 147.

[2] N. N. Dinh, D. N. Chung, T. T. Thao, T. T. Chung Thuy, L. H. Chi, T. Vo-Van, Enhancement of performance of organic light emitting diodes by using Ti- and Mo-oxide nano hybrid layers, Mater. Sci. Appl., 4 (2013) 275.

[3] N. N. Dinh, T. T. Thao, D. N. Chung, V.-V. Truong, Characterization of organic solar cells made from hybrid photoactive materials of P3HT:PCBM/nc-TiO 2 , VNU Journal of Mathematics - Physics, 30 (2014) 8.

[4] M.H. Hoang, T.T. Dao, N.T.T. Trang, P.H.N. Nguyen, T.T. Ngo, Synthesis of gold nanoparticles capped with quaterthiophene for transistor and resistor memory devices, Journal of Chemistry, 2016 (2016) 8 pages.

[5] Y. Wakayama, R. Hayakawa, H.-S. Seo, Recent progress in photoactive organic field-effect transistors, Sci. Technol. Adv. Mater. 15, (2014) 024202.

[6] http://www.eoc-inc.com/UV_detectors_silicon_carbide_photodiodes.htm, accessed 16 March 16, 2017.

[7] S.R. Forrest, Active optoelectronics using thin-film organic semiconductors, IEEE J. Select. Topics Quantum Electron. 6 (2000) 1072.

[8] T.D. Anthopoulos, Electro-optical circuits based on light-sensing ambipolar organic field-effect transistors, App. Phys. Lett. 91 (2007) 113513.

[9] J.-M. Choi, J. Lee, D. K. Hwang, J. H. Kim, S. Im, Comparative study of the photoresponse from tetracene-based and pentacene-based thin-film transistors, Appl. Phys. Lett. 88 (2006) 043508.

[10] B. Lucas, A. El Amrani, M. Chakaroun, B. Ratier, R. Antony, A. Moliton, Ultraviolet light effect on electrical properties of a flexible organic thin film transistor, Thin Solid Films, 517 (2009) 6280.

[11] El Amrani, B. Lucas, F. Hijazi, A. Skaiky, T. Trigaud, M. Aldissi, Transparent pentacene-based photoconductor: high photoconductivity effect, Eur.Phys. J. Appl. Phys. 51 (2010) 33207.

[12] A. El Amrani, B. Lucas, B. Ratier, The effect of the active layer thickness on the performance of pentacene-based phototransistors, Synthetic Metals, 161 (2012) 2566.

[13] D. Yang, L. Zhang, S. Y. Yang, B. S. Zou, Influence of the dielectric PMMA layer on the detectivity of pentacene-based photodetector uith field-effect transistor configuration in visible region, IEEE Photonics Journal, 5 (2013) 6801709.

[14] T. T. Dao, T. Matsushima, M. Murakami, K. Ohkubo, S. Fukuzumi, and H.Murata, Enhancement of ultraviolet light responsivity of a pentacene photoOTFT by introducing photoactive molecules into a gate dielectric, Jpn. J. Appl.Phys., 53 (2014) 02BB03.

[15] S.-H. Yuan, Z. Pei, H.-C. Lai, C.-H. Chen, P.-W. Li, Y.-J. Chan, Au nanoparticle light scattering enhanced responsivity in pentacene phototransistor for deep-UV light detection, IEEE Electron Device Letters, 36 (2015) 1186. 